\title{
IL-2 overrules tolerogenic liver responses
}

Antigen-presenting cells (APCs) in the liver generally induce $\mathrm{T}$ cell tolerance rather than $\mathrm{T}$ cell immunity. Of the APCs in the liver, liver sinusoidal endothelial cells (LSECs) are highly adept at taking up soluble antigens from the blood and cross-presenting these antigens on MHC class I molecules to passing naive $\mathrm{CD} 8^{+} \mathrm{T}$ cells. This antigenspecific interaction invariably induces tolerogenic $\mathrm{T}$ cells through

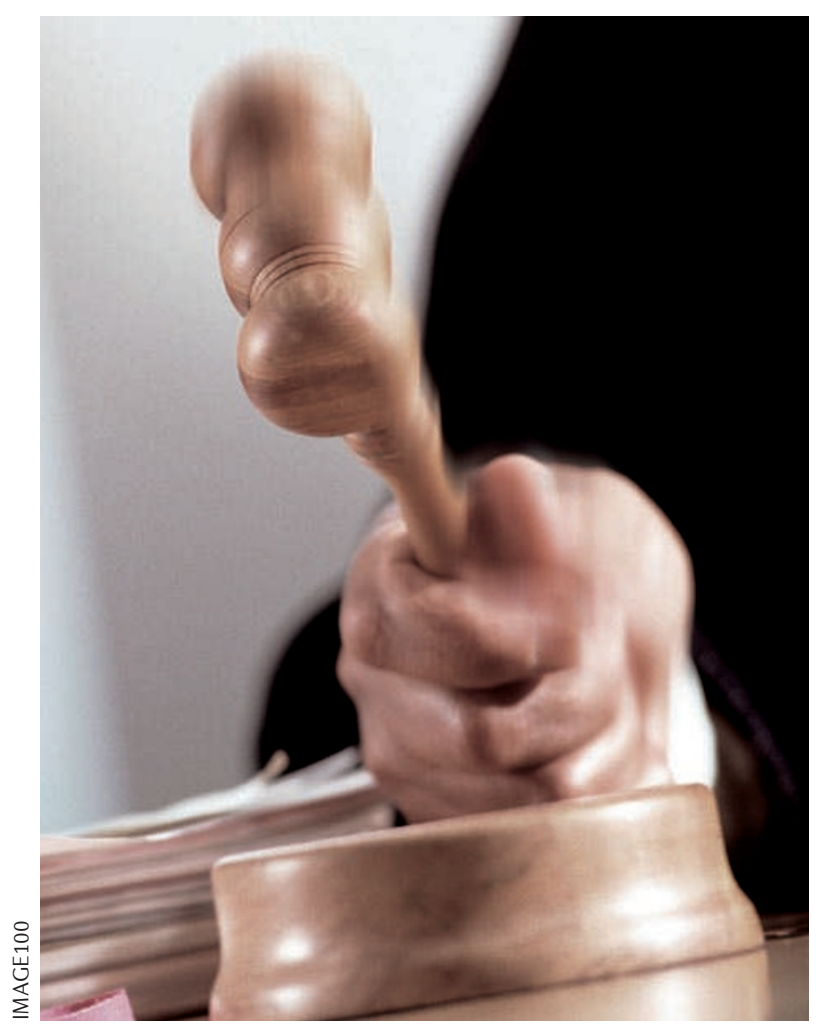

a mechanism involving upregulation of the inhibitory molecule B7-H1 (also known as PDL1) on LSECs and programmed cell death 1 (PD1) on the T cells. Given the known importance of duration and strength of T cell receptor (TCR) signalling for the differentiation, proliferation, function and tolerance of T cells, Knolle and colleagues investigated whether the number of peptideloaded MHC class I molecules on cross-presenting LSECs influences tolerogenic or immunogenic $\mathrm{T}$ cell priming in the liver.

First, the authors established that LSECs incubated with increasing concentrations of the model antigen ovalbumin (OVA) led to a concomitant rise in the number of OVA peptide-loaded MHC class I molecules on their surface. They then showed that at low antigen concentrations $(10-100 \mu \mathrm{g} / \mathrm{ml})$, LSECs induced tolerance of naive OVA-specific $\mathrm{CD}^{+} \mathrm{T}$ cells but at high antigen concentrations $(1 \mathrm{mg} / \mathrm{ml})$ the T cells were not tolerized, as they responded to restimulation with the expression of interferon- $\gamma(\mathrm{IFN} \gamma)$ and interleukin-2 (IL-2).

Next, the authors investigated the mechanism of loss of tolerance induction by LSECs exposed to high concentrations of antigen. The effect was not dependent on upregulated expression of the co-stimulatory molecules CD80 or CD86, as LSECs lacking these molecules still generated IFN $\gamma$-producing effector T cells.
Instead, early IL-2 production by the $T$ cells during their initial contact with LSECs was found to be responsible for overriding the tolerogenic response. Functional inactivation of IL- 2 by neutralizing antibodies prevented the induction of IFN $\gamma$ producing $\mathrm{T}$ cells, whereas addition of exogenous IL-2 during T cell priming by LSECs under otherwise tolerogenic conditions allowed the development of effector function.

The authors also found a direct correlation between antigen concentration and expression of B7-H1 on LSECs: low antigen levels were associated with increased B7-H1 expression, whereas its expression was curtailed at high antigen levels. The finding that $\mathrm{T}$ cells stimulated by B7-H1-deficient LSECs released more IL-2 than those primed by wild-type LSECs and differentiated into effector T cells supports the hypothesis that B7-H1-mediated signals limit IL-2 production induced by TCR signalling.

This IL-2-dependent mechanism for overriding tolerance in the presence of high antigen levels, but the absence of CD80 or CD86 co-stimulation, may be important for inducing $\mathrm{T}$ cell responses during viral infection in the liver.

Lucy Bird

ORIGINAL RESEARCH PAPER Schurich, A. et al. Dynamic regulation of $\mathrm{CD} 8 \mathrm{~T}$ cell tolerance induction by liver sinusoidal endothelial cells. J. Immunol. 184, 4107-4114 (2010) 